\title{
Simulated propagation of ultrashort pulses modulated by low-Fresnel-number lenses using truncated series expansions
}

\author{
Ronan J. Mahon* and J. Anthony Murphy \\ Department of Experimental Physics, National University of Ireland, Maynooth, Co. Kildare, Ireland \\ ${ }^{*}$ Corresponding author: ronanjmahon@gmail.com
}

Received 3 April 2014; revised 14 July 2014; accepted 18 July 2014;

posted 21 July 2014 (Doc. ID 209472); published 27 August 2014

\begin{abstract}
Numerical simulation of the paraxial propagation of pulses modulated by lenses is demonstrated using the Laguerre-Gaussian (LG) series expansion method. This technique allows for relatively swift evaluation of the structures of several individual monochromatic fields transformed by arbitrary amplitude and phase modulating pupil functions, which can be superimposed via the inverse Fourier transform to determine the structure of a modulated pulse. The transformation of ultrashort pulses by spherical, diffractive, and conical lenses is simulated using this method, which is particularly effective with the use of vector and matrix techniques available in many popular numerical software packages. A description of the convergence of the LG series to the results of the conventional integral techniques is presented for a conical lens under illumination by a continuous wave from which a simple but robust criterion for axial accuracy in problems of circular symmetry is suggested. (c) 2014 Optical Society of America

OCIS codes: $\quad$ (320.5550) Pulses; (050.1965) Diffractive lenses; (070.7345) Wave propagation.

http://dx.doi.org/10.1364/AO.53.005701
\end{abstract}

\section{Introduction}

Analytical expressions for fundamental optical beams can be derived in many cases, but the propagation of waves modulated by apertures or elements with irregular structures can require numerical techniques for accurate description. In the paraxial regime, simulation of wave propagation within optically large regions relies heavily on the use of the Fresnel diffraction integral (FDI) [1] . Computational solution of the FDI in its basic form is inherently a relatively intensive calculation and, despite the availability of cheap and fast computing power, prediction of $2 \mathrm{D}$ field structures may require several hours or longer. Often, as with the derivation of analytical expressions, application of the FDI is restricted to the calculation of fields on the optical axis only.

$1559-128 \mathrm{X} / 14 / 255701-11 \$ 15.00 / 0$

(C) 2014 Optical Society of America
Approximation of the FDI using asymptotic series expansions has been explored and offers a useful method of simulating the propagation of paraxial waves. One example, initially applied in acoustics, is Wen and Breazeale's method that allows for efficient description of paraxial diffraction effects using series with as few as 10 terms [2]. However, a drawback in the original description of this technique is that the calculation of the series coefficients for the Gaussian beam basis terms relies on search algorithms, thereby prohibiting a fast and impromptu analysis of arbitrary apertures. Ding and Zhang described a more efficient procedure for calculating the expansion coefficients in that method, but their algorithm is noted to remain stable only for a series with less than approximately 50 expansion terms [3]. Zamboni-Rached and co-workers have recently formulated the propagation of a diffracted field as an expansion in Bessel-Gauss beams [4,, 5$]$, illustrating the potential efficiency of the series expansion techniques with high-resolution 2D field structures 
calculable in seconds. Although such expansion methods will always exhibit a reduced accuracy from that of the FDI through the exclusion of higher-order series terms and the related spatial frequencies, the loss of information can be negligible. These methods merit application through their ability to utilize vector and matrix arithmetic techniques found in many numerical software packages, such as MATLAB/Octave, Python/Numpy, etc., allowing for the manipulation of transverse field structures on a plane-byplane basis $[6,7]$ rather than the more time consuming point-by-point scheme required for solution of the FDI in its fundamental form. The conventional concept of an optical pulse as an integration of monochromatic fields weighted according to the Fourier transform of the modulating signal can require the calculation of dozens or hundreds of field calculations, each related to an individual spectral component. The efficiency of the series expansion techniques in calculating monochromatic fields can significantly reduce the time required for simulation of pulses from that of the FDI.

Here, extending the work of $[\underline{6}, 7]$, we describe the application of the Laguerre-Gaussian (LG) expansion [8-15] in simplifying the calculation of pulses transformed by phase modulating elements. Within this discussion the convergence of the LG calculations to those of the FDI is illustrated for a conical lens, with the suggestion of a simple but effective estimate for the minimum number of series terms required for accurate field calculations on the optical axis.

\section{A. Laguerre-Gaussian Series Expansion}

The LG beams are eigenmode solutions of the paraxial wave equation and are described in cylindrically symmetric $r-z$ space as $[\underline{7,} \underline{10}]$

$$
\begin{aligned}
\psi_{m}(\omega, r, z)= & \sqrt{\frac{2}{\pi w^{2}(z)}} L_{m}\left(\frac{2 r^{2}}{w^{2}(z)}\right) \exp \left(-\frac{r^{2}}{w^{2}(z)}\right) \\
& \times \exp \left(i \frac{\omega}{c}\left(z+\frac{r^{2}}{2 R(z)}\right)\right) \\
& \times \exp \left(-i(2 m+1) \tan ^{-1}\left(\frac{2 c z}{\omega w_{0}^{2}}\right)\right),
\end{aligned}
$$

where $L_{m}$ is the Laguerre polynomial with integer index $m$.

The variation of the beam width parameter is

$$
w(z)=w_{0} \sqrt{1+\left(\frac{2 c z}{\omega w_{0}^{2}}\right)^{2}}
$$

with $w_{0}=w(0)$, which is an important parameter in this work. The radius of phase curvature of the wavefront is

$$
R(z)=z\left(1+\left(\frac{\omega w_{0}^{2}}{2 c z}\right)\right)
$$

and the confocal range is given by

$$
z_{c}=\frac{\omega w_{0}^{2}}{2 c}=\frac{\pi w_{0}^{2}}{\lambda}
$$

with angular frequency, $\omega$, wavelength, $\lambda$, and speed of light, $c$. The effective radii of the LG beam modes are $[16]$

$$
\rho(z)=w(z) \sqrt{2 m+1} .
$$

The paraxial propagation of a continuous wave (CW) transformed by a phase modulating aperture or lens with a frequency dependent pupil function, $E_{0}\left(\omega, r_{0}\right)$, can be approximated as a truncated series of $M+1$ LG beam modes $\psi_{m}$ from

$$
E_{\mathrm{CW}}(\omega, r, z)=\sum_{m=0}^{M} A_{m}(\omega) \psi_{m}(\omega, r, z),
$$

with the expansion coefficient, $A_{m}(\omega)$, determined as

$$
\begin{aligned}
A_{m}(\omega)= & \sqrt{\frac{2}{\pi w_{0}^{2}}} \int_{0}^{a} E_{0}\left(\omega, r_{0}\right) L_{m}\left(2 \frac{r_{0}^{2}}{w_{0}^{2}}\right) \\
& \times \exp \left(-\frac{r_{0}^{2}}{w_{0}^{2}}\right) 2 \pi r_{0} \mathrm{~d} r_{0},
\end{aligned}
$$

where $a$ is the radius of the aperture and $r_{0}$ is the radial coordinate at the plane of the aperture, which is assumed here to lie at $z=0$. Equation (4) can also be applied as an overlap integral using the complex conjugate of the LG beams in Eq. (1) with the $R(z)$ term adapted to account for the phase curvature of a field [14]. Here, to generalize the procedure, we will discuss the expansion method with application of the fundamental LG functions, as in Eq. (4), in reference to its potential use without prior knowledge of the optical parameters of a beam or lens.

From the recurrence relations of the Laguerre polynomials, basic expressions can be derived for calculation of the expansion coefficients, $A_{m}$, of a circular aperture illuminated by a plane wave $[8,11]$ or higher order LG beams [12]. With these relations, the expansion of zone plates can be simplified through their representation as a superposition of positive and negative circ functions of appropriate radii [13], an approach which could potentially be extended to the modeling of diffractive Fresnel lenses. However, the application of such recurrence relations may be limited to very specific and ideal beams/apertures only and, as with solution of the FDI, it is perhaps not efficient to attempt to derive analytical expressions for every aperture/beam profile that may be suspected to be tractable. It is suggested that the most significant benefit of the Gaussian expansion methods is in their application as numerical tools.

For an $M+1$ term expansion of a circular aperture the optimum value of $w_{0}$ is given by [8] 


$$
w_{0}=\frac{a}{\sqrt{M}},
$$

which is applied throughout this work. We represent the median integer index of a series as $m=\mu$ and with the application of an odd value of $M$ in this work $\mu=(M-1) / 2$, corresponding to a radius at the aperture plane of $\rho_{\mu}(0)=a$.

The expansion of a propagating field in terms of LG or Hermite-Gaussian (HG) beam modes can allow for efficient asymptotic calculation of fields within the paraxial regime, which, for the simulation of multispectral pulses, can considerably reduce computation times without a significant loss of accuracy $[6,7]$. For a pulsed wave, $E_{\mathrm{PW}}(r, z, t)$, the field structures are predicted through an inverse Fourier transform representing an integration of monochromatic waves at varying frequencies, i.e.,

$$
E_{\mathrm{PW}}(r, z, t)=\frac{1}{\sqrt{2 \pi}} \int_{-\infty}^{\infty} S(\omega) E_{\mathrm{CW}}(\omega, r, z) e^{-i \omega t} \mathrm{~d} \omega,
$$

where $S(\omega)$ is the Fourier transform of the timevarying amplitude modulation of the pulsed field, $s(t)$. Expanding each spectral field, $E_{\mathrm{CW}}(\omega, r, z)$, as in Eq. (3), the propagation of a pulsed wave is then determined from [7]

$$
\begin{aligned}
& E_{\mathrm{PW}}(r, z, t) \\
& \quad=\frac{1}{\sqrt{2 \pi}} \int_{-\infty}^{\infty} S(\omega)\left\{\sum_{m=0}^{M} A_{m}(\omega) \psi_{m}(\omega, r, t) e^{-i \omega t}\right\} \mathrm{d} \omega .
\end{aligned}
$$

With no phase modulation across an aperture, the values of $A_{m}$ are independent of angular frequency, $\omega$, and the sum and integral in Eq. (7) can be rearranged, allowing a pulse to be conceptualized, as in Ref. [7], as a superposition of individual LG "pulselets." Within such a description, the behavior of the individual pulselets can be related to the physical features of pulse diffraction at an aperture; in particular, the propagation of the boundary waves generated at the edge of the aperture $[17,18]$. However, the description in Ref. [7] was based on the numerical simulation of the pulselets; therefore, not enabling the potential efficiency of the expansion technique that could be obtained with closed expressions for the individual pulsed modes. Analytical formulation of the propagation of the LG pulselets will require the derivation of expressions for each unique spectral variation, $S(\omega)$, but Martínez-Matos et al. have recently augmented the potential of the LG expansion in pulse modeling with the derivation of a formula for the propagation of the LG pulselets with Gaussian spectra [19]. In contrast to [7] and [19], the LG expansion method is discussed here through the conventional model of a pulse as an integration of monochromatic fields [6], as in Eq. (7), illustrating the technique as an efficient alternative to the FDI. For pulses transformed by lenses, the concept of an ultrashort field propagating as a superposition of individual LG pulselets [7,19] would remain valid, with each individual pulselet subject to modulation. However, the spectral dependence of the mode coefficients, $A_{m}(\omega)$, in Eq. (7) is suggested to add significant complexity to the potential derivation of closed expressions for the LG pulselets influenced by dispersion and phase modulation.

The following section briefly illustrates the application of the LG expansion in simulating pulses modulated by diffractive forms of the spherical and Fresnel lenses. In Section 3, details of the expansion of a conical lens are given that will also relate to the general application of the method for spherical lenses and apertures.

\section{Simulation of Diffractive Lenses Using the Laguerre-Gaussian Expansion Method}

Diffractive Fresnel lenses, e.g., [20-23], are primarily suited as a method of concentrating energy, but can also be used for image formation [24]. The focusing effect of any lens on a wave is dependent on the frequency of the field, which can create complex propagation features in pulses [25-27], thus, requiring numerical methods for accurate descriptions. The illustration here of the LG expansion as an alternative procedure for simulating lens-modulated pulses is a relevant topic, with recent technological developments enabling high resolution measurements $[28,29]$ or "snapshots in flight" [30] of ultrashort fields using interferometric methods. Alongside the progress of such experimental work, the intuitive boundary wave theory of pulse diffraction has been applied and augmented in the analysis of optical elements [17,18,31-34], allowing in some cases for the derivation of concise and accurate analytical expressions of pulse structures [34].

Examples of calculations using the LG expansion will be given in this section for the propagation of pulses modulated by quantized forms of spherical and Fresnel lenses, with the ideal lens surfaces reduced to $P$ discrete levels. A diffractive form of the spherical lens, the "staircase" kinoform, is constructed by quantizing the height of a thin spherical lens to a constant value within each Fresnel zone such that $P=N_{F}$, where $N_{F}$ is the Fresnel number of the lens [20]. Also, to illustrate the variability of the expansion method for analyses of unorthodox lens profiles, we simulate the propagation of a pulse modulated by a simple modification of a thin lens with the spherical profile approximated as a finite number of linear segments, with such an element referred to here as a linear surface lens. The profiles of these lenses are illustrated in Fig. 1, with the radial variation of the lens thickness, $h(r)$, shown relative to the maximum thickness, $h_{0}$, of the ideal lens forms. Due to dispersion, the focal length and Fresnel number of a lens will vary with frequency; therefore, the lenses are described in relation to pulses through the Fresnel number, $N_{0}=a^{2} /\left(\lambda_{0} f_{0}\right)$, with the wavelength, $\lambda_{0}$, and focal length, $f_{0}$, at the pulse's carrier 

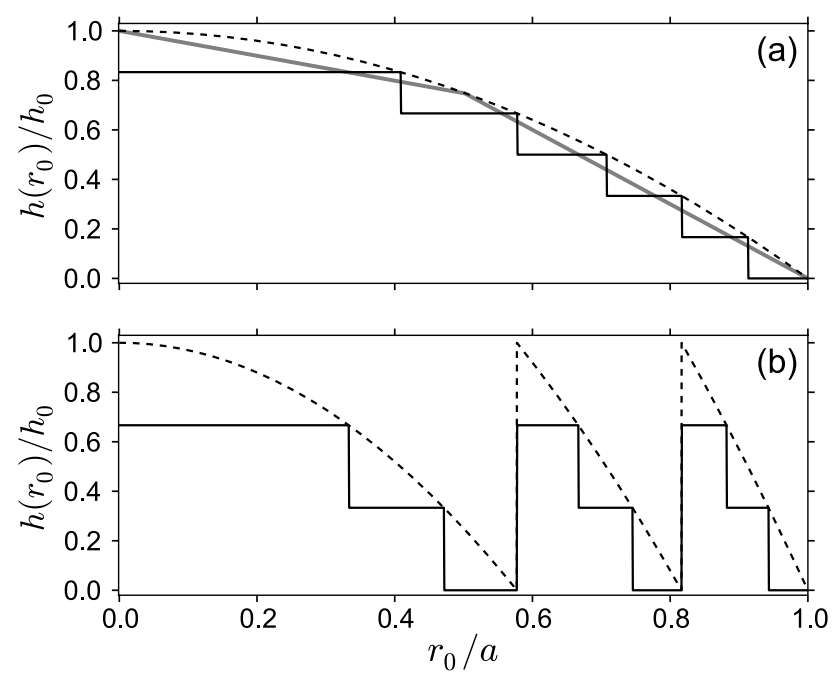

Fig. 1. Structure of the types of lenses simulated in this paper with a Fresnel number of $N_{0}=6$. (a) Quantized $P=6$ kinoform (solid lines) with a linear surface lens (gray) approximating a thin spherical lens (dashed curve), and (b) diffractive Fresnel lens with $P=3$ (solid lines) and the ideal Fresnel lens (dashed curves).

frequency. In this work, the carrier frequency corresponds with the design frequency of the lenses.

The pulses are taken to be modulated in time, $t$, by a conventional Gaussian signal,

$$
s(t)=\exp \left(-\ln 2 \frac{t^{2}}{\tau_{0}^{2}}\right) \exp \left(-i \omega_{0} t\right)
$$

with a full width at half-maximum (FWHM) of $2 \tau_{0}$ and a carrier angular frequency of $\omega_{0}$. The Fourier spectrum of the signal in Eq. () is

$$
S(\omega)=\frac{\tau_{0}}{\sqrt{2 \ln 2}} \exp \left(-\tau_{0}^{2} \frac{\left(\omega-\omega_{0}\right)^{2}}{4 \ln 2}\right),
$$

with a fractional bandwidth $\gamma=\Delta \omega / \omega_{0}$, where $\Delta \omega=$ $4 \ln 2 / \tau_{0}$ is the FWHM of the spectrum.

The phase modulation of a thin lens with refractive index $n(\omega)$ is represented as [1]

$$
\phi\left(\omega, r_{0}\right)=i \frac{\omega}{c}(n(\omega)-1) h\left(r_{0}\right)+i \frac{\omega}{c} h_{0},
$$

and the mode coefficients, $A_{m}(\omega)$, are calculated from Eq. (4), with $E_{0}\left(\omega, r_{0}\right)=\exp \left(i \phi\left(\omega, r_{0}\right)\right)$, assuming here that the lenses are illuminated uniformly by a plane wave. Figure $\underline{2}$ illustrates the variation of the mode coefficients at each angular frequency for the described lenses. The coefficients are illustrated as $\operatorname{Re}\left\{A_{m}(\omega) /(-1)^{m}\right\} / w_{0}(2 \pi)^{1 / 2}$ [7] and are modulated by the normalized variation of the Gaussian spectrum, $S(\omega) / S\left(\omega_{0}\right)$. The images shown in Fig. 2 represent the fundamental data required for the pulse simulations to be illustrated in this work.

Calculated using an LG expansion with $M=499$, Figs. 3 and 4 illustrate the propagation of pulses modulated by the described lenses. The $\mathrm{SiO}_{2}$

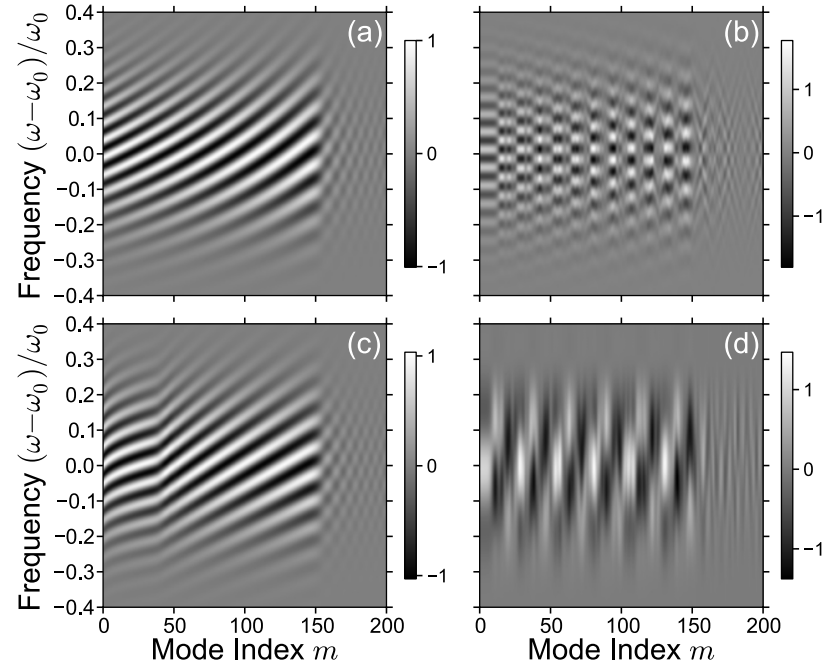

Fig. 2. Normalized LG mode coefficients, $\operatorname{Re}\left\{A_{m}(\omega) /(-1)^{m}\right\}$, calculated from Eq. (4) for a $\gamma=0.3$ plane wave pulse modulated by various lenses with $N_{0}=12$, using $M=299$. (a) Spherical lens, (b) staircase kinoform, (c) linear surface lens, and (d) $P=3$ diffractive Fresnel lens. The data are modulated vertically by the normalized structure of the pulse spectrum, $S(\omega)$.

microlenses simulated here have a focal length of $f_{0}=8 \mathrm{~mm}$ at the design wavelength $\lambda_{0}=800 \mathrm{~nm}$, and with a Fresnel number of $N_{0}=12$ have a diameter of $0.55 \mathrm{~mm}$. A large pulse bandwidth of $\gamma=0.3$ is applied to enhance propagation features, and the temporal (vertical) axes are related to the time delay $z / c+h_{0} / v_{g}$ where $v_{g}$ is the group velocity within the lens medium. With a low Fresnel number, the fields in Figs. 3 and $\underline{4}$ are not subject to a significant level of material dispersion by the lens, which is illustrated by the temporal consistency of the focal spot radii of the pulses modulated by the spherical lens and its derivatives shown in Figs. 3(e), 3(j) and 4(e). In Fig. $4(\mathrm{j})$ the considerable effects of a spatial dispersion induced by the Fresnel form of lens can be seen with the focal length of each spectral component altered according to $f(\omega)=\omega f\left(\omega_{0}\right) / \omega_{0}$, and the spot radius at the focal plane is observed to decrease in time. This is in contrast to pulses subject to large group velocity dispersion effects by spherical lenses for which the spot radius at the focal plane often increases in time $[\underline{25}, \underline{26}]$.

\section{Laguerre-Gaussian Expansion of Pseudo-Bessel Beams Generated by Axicons}

The conical lens, or axicon, has become a more familiar element in optical studies in the last decade or so [35-39]. The axicon projects all perpendicularly incident rays at an equal angle toward the optical axis, forming a focal line that maintains a consistent spot size over a significant distance, $L$, often referred to as the diffraction free range, see Fig. $\underline{5}$. The refraction of a CW plane wave by a conical lens generates a physical approximation of the Bessel beam, $J_{0}(\alpha r) \exp (i \beta z-i \omega t)$, where $\alpha^{2}+\beta^{2}=k^{2}$ with wavenumber, $k=\omega / c$ [40]. Under pulsed illumination these lenses create a practical form of the fields 

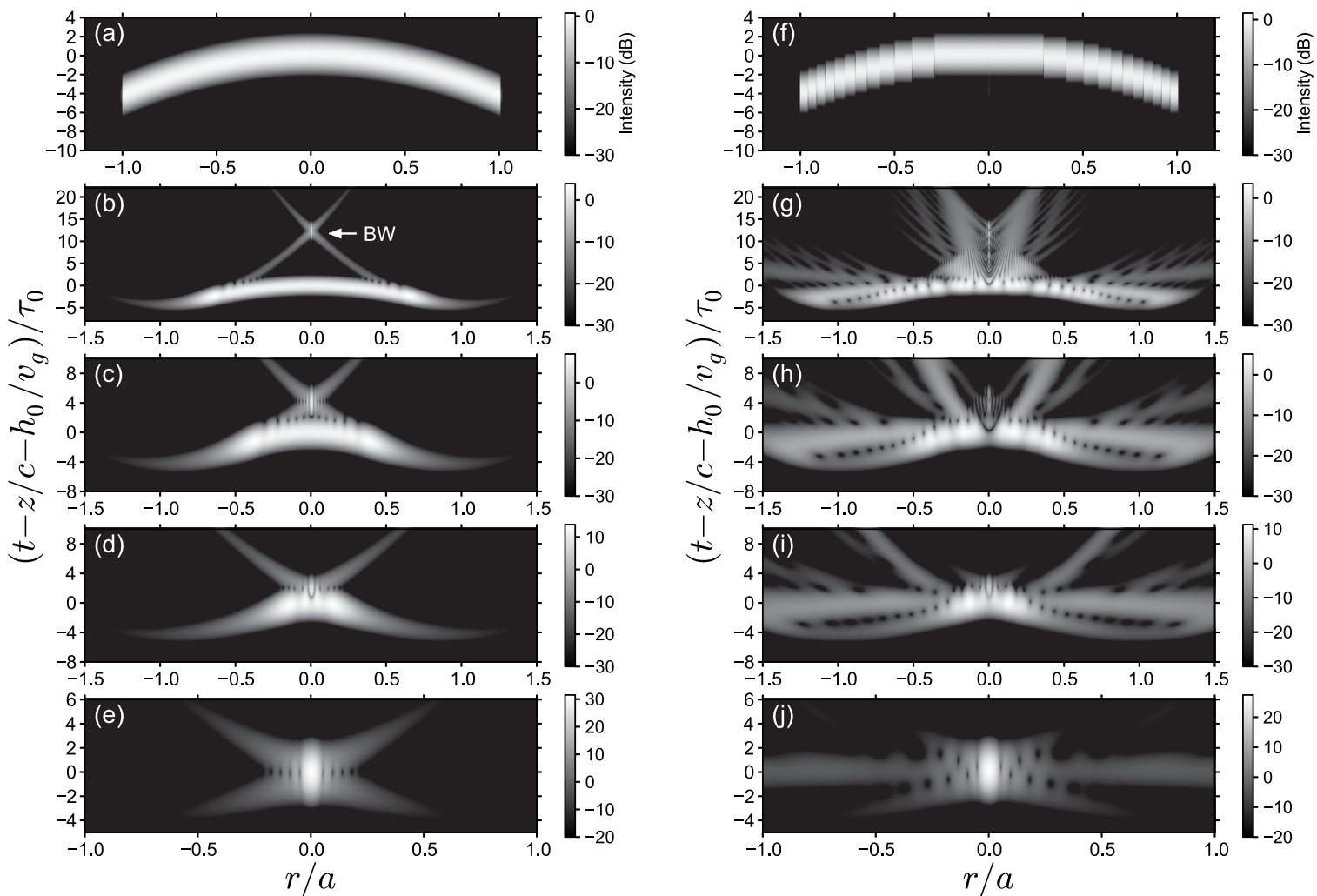

Fig. 3. Propagation of a $\gamma=0.3$ pulse modulated by a (left) thin spherical lens and (right) a staircase kinoform lens with $P=N_{0}=12$. The fields are calculated at $z=(\mathrm{a})$ and (f) 0 , (b) and (g) $f_{0} / 4$, (c) and (h) $f_{0} / 2$, (d) and (i) $3 f_{0} / 4$, (e) and (j) $f_{0}$. "BW" in (b) distinguishes the boundary wave from the primary geometric wave [17].
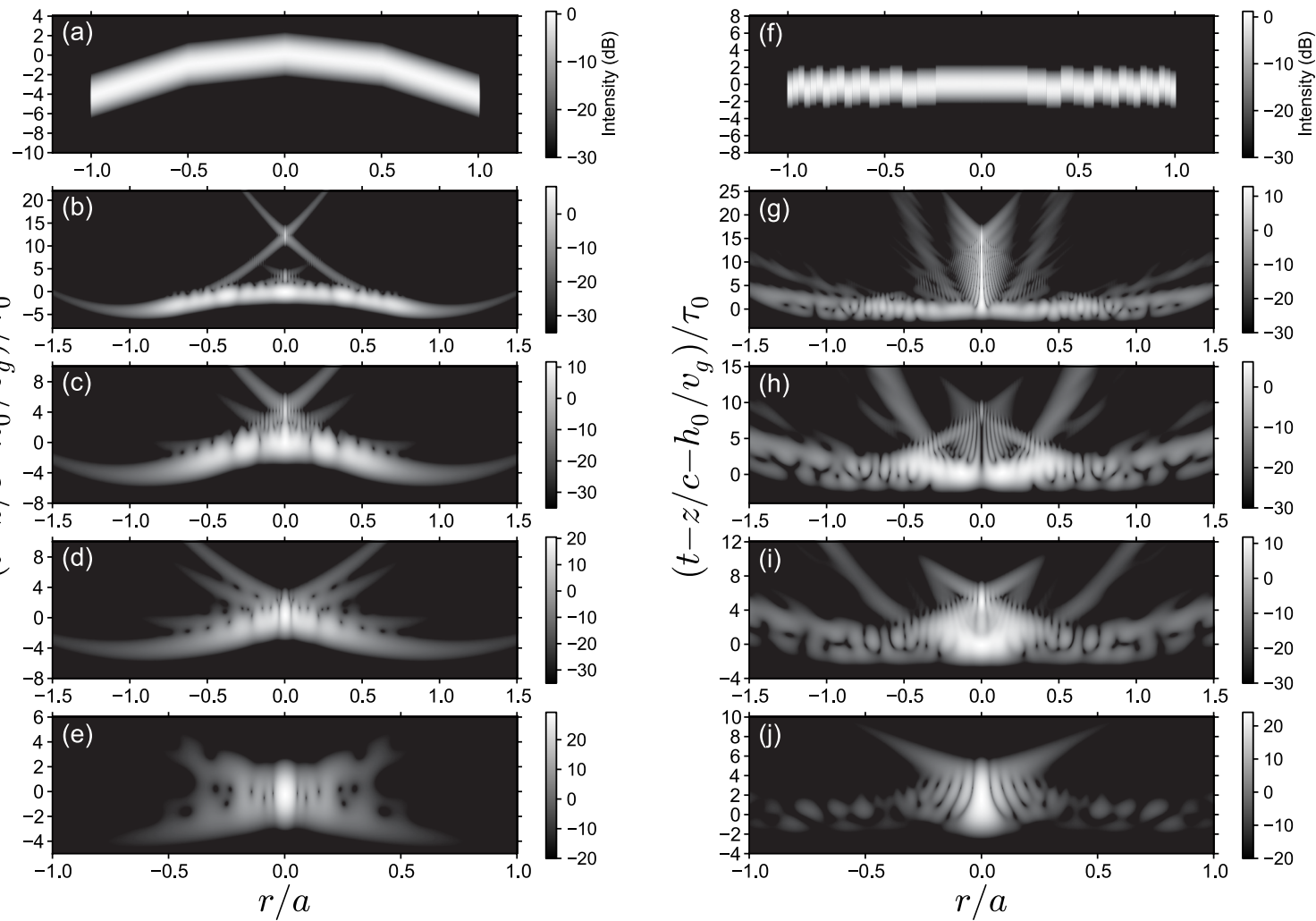

Fig. 4. $\gamma=0.3$ pulse transformed by a (left) linear surface lens and (right) a diffractive Fresnel lens with $P=3$, both with $N_{0}=12$. The fields are calculated at $z=(\mathrm{a})$ and (f) 0 , (b) and (g) $f_{0} / 4$, (c) and (h) $f_{0} / 2$, (d) and (i) $3 f_{0} / 4$, (e) and (j) $f_{0}$. 


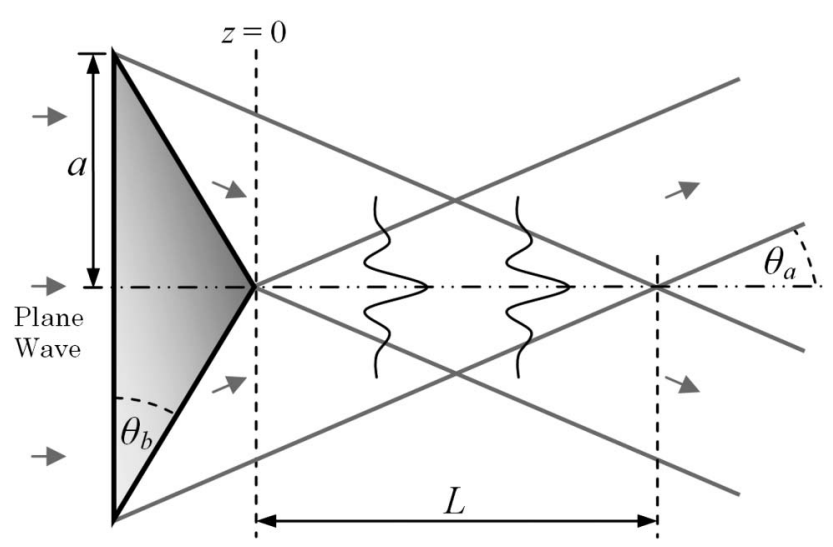

Fig. 5. Generation of a pseudo-Bessel beam or focal line on the optical axis between $z=0$ and $z=L$ by the self-interference of a plane wave refracted by an axicon.

known as $\mathrm{X}$ waves $[30, \underline{41}-\underline{43}]$. A previous description of the LG expansion for conical lenses is given in Ref. [36], which is extended upon here.

The thickness of a conical lens is $h\left(r_{0}\right)=h_{0}-$ $r_{0} \tan \theta_{b}$, with a maximum of $h_{0}=a \tan \theta_{b}$ for an axicon base angle of $\theta_{b}$, and the phase modulation can be represented as in Eq. (10). For a CW the Fresnel number of a conical lens has a similar form to that of the conventional thin lens, given as [37]

$$
N_{F}=\frac{a^{2}}{\lambda L}
$$

where $L$ is the geometric range of the focal line generated,

$$
L=a \beta / \alpha .
$$

$\alpha$ and $\beta$ are the radial and longitudinal components of the wavenumber, $k$, calculated as

$$
\begin{aligned}
& \alpha=k \sin \theta_{a}, \\
& \beta=k \cos \theta_{a},
\end{aligned}
$$

and $\theta_{a}$ is the angle of refraction with respect to the optical axis, predicted geometrically from Snell's law as

$$
\theta_{a}=\sin ^{-1}\left(n \sin \theta_{b}\right)-\theta_{b} \approx(n-1) \theta_{b} .
$$

In the simulation of pulses these beam parameters will vary for different frequencies due to dispersion of the lens medium with $n(\omega)$.

The radial intensity profile of a plane wave focused by an axicon is proportional to $J_{0}(\alpha r)^{2}$ near the axis, while the axial $(r=0)$ irradiance increases linearly with propagation distance. In the geometric regime the on-axis intensity can be represented as [39]

$$
I(z)=4 \pi^{2} z N_{F} \operatorname{rect}\left(\frac{z}{L}-\frac{1}{2}\right) / L,
$$

where $\operatorname{rect}(x)=1$ for $|x|<1 / 2$ and 0 for all other values. With relatively low Fresnel numbers applied in this work, diffraction effects will greatly effect the validity of Eq. (15), but it serves as a useful summary of the axial properties of an axicon beam.

\section{A. LG Synthesis of the Conical Lens}

In describing the LG expansion of a conical lens for CWs, as a comparison we recall that Fourier transformation of the phase representation of a thin spherical lens with a focal length, $f, \exp \left(-i k r^{2} / 2 f\right)$, shows a wide spectral range of radial frequencies, $k_{r}$, dominant within $k_{r} \approx \pm k a / f$ and inclusive of the axial ray with $k_{r}=0$. Similarly, in a LG expansion of the thin lens function, the coefficients of lower order modes with $m \rightarrow 0$ maintain importance, coupling with the low frequency components, $k_{r} \rightarrow 0$ of the lens toward the optical axis. However, in the LG decomposition of a thin axicon's phase function, with constant radial frequency, $k_{r} \approx \alpha$, it is observed that the values of $A_{m}$ for modes of lower indices can be significantly reduced. In such cases, appreciable coefficients are maintained only by the higher-indexed LG functions with radial frequency spectra inclusive of the axicon beam's radial frequency, $\alpha$. The extent of the Fourier-Bessel spectrum of an LG mode, corresponding to its radial frequency at $r \rightarrow 0$, can be approximated as [7]

$$
\kappa_{m}(z)=\frac{2}{w(z)} \sqrt{2 m+1} .
$$

With $w(0)$ from Eq. (5), an estimate for the minimum index of the modes with Fourier-Bessel spectra inclusive of the radial frequency of the axicon beam, i.e., $\kappa_{m}(0) \geq \alpha$, is

$$
\Delta m=\left[\frac{\alpha^{2} a^{2}}{8 M}-\frac{1}{2}\right],
$$

which can be seen in the following Fig. 6 to relate to a shift of the axicon's spectrum of $L \bar{G}$ coefficients, akin to an offset of its Fourier spectrum. In Fig. 6(a) the predicted modal shift is $\Delta m=6$ and in (b) $\Delta m=40$. For expansion of the corresponding spherical lenses, a stretching of the LG spectra is observed with a reduction of the coefficient values preserving the constant sum of $\left|A_{m}\right|^{2}$ over $m$ for each aperture function. In the simulation of modulated pulses, these features will be emphasized for higher field frequencies.

Equation ( $\underline{5}$ ) is an optimum value for expansion of the circular aperture because it creates a balance between the number of LG functions that are significantly obstructed at $r_{0}=a\left(\rho_{m}>a\right)$, and those that are not $\left(\rho_{m} \leq a\right)$, thus placing equal emphasis on the synthesis of both the gross structure and the discontinuity at $r_{0}=a[7,8]$. With the radial truncation of the modes with indices $m>\mu\left(\rho_{m}>a\right)$, the corresponding coefficient values are significantly reduced, see Fig. $\underline{6}$ with $\mu=149$. However, for the 

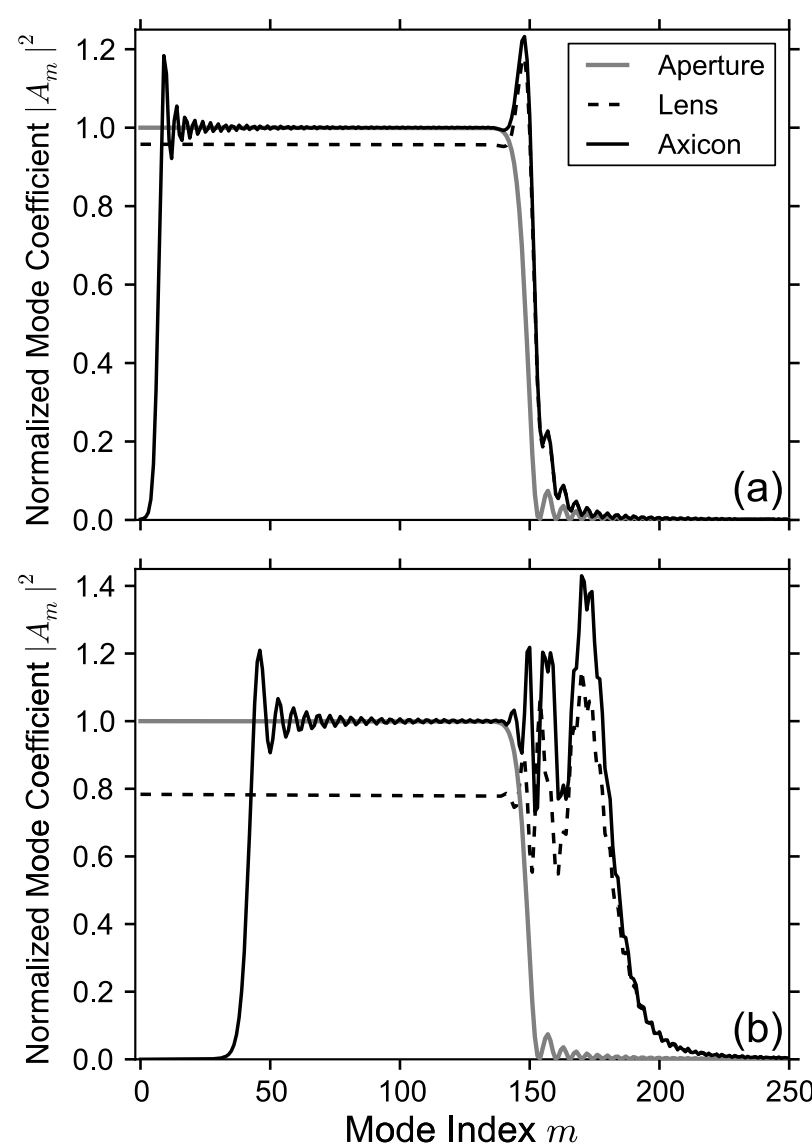

Fig. 6. LG coefficients from Eq. (4), with $M=299$, for a circular aperture and conical and spherical lenses with a Fresnel number, $N_{F}=$ (a) 20 , and (b) 50 .

altered spectra of conical or spherical lenses, many coefficients with $m>\mu$ are seen to retain appreciable values despite the radial truncation of the modes. This is possible due to the maintenance of a correlation between the structures of the respective LG functions and the oscillatory lens functions at $r_{0} \rightarrow a$. Determination of the modes that preserve such correspondence cannot, to the authors' knowledge, be achieved in a direct manner, as the local frequencies of the LG functions are not formally identifiable, for example, as $\kappa_{m}(r)$. However, for thin conical and spherical lenses, using $\alpha \approx k a / f$ for the latter, $\Delta m$ from Eq. (17) is observed as a valid estimate for the extension of the modal spectra beyond $m=\mu$.

Despite the deviations of the LG spectra of lenses shown in Fig. 6 from that of the circular aperture, it is important to note that the width parameter defined by Eq. (5) is valid for optimal expansions of the specific lens functions described here, and will remain so when a sufficiently large value of $M$ is employed. This is shown in Fig. 7, with a comparison of the normalized mean squared error $\Delta E_{0}$ between lens/aperture functions and their respective syntheses using the LG expansion $[8,10,15]$.

In Fig. $\underline{7}$, the value of $w_{0}$ predicted with Eq. (5) is seen to remain valid for optimal synthesis of the axicon, lens, and circular aperture, minimizing the

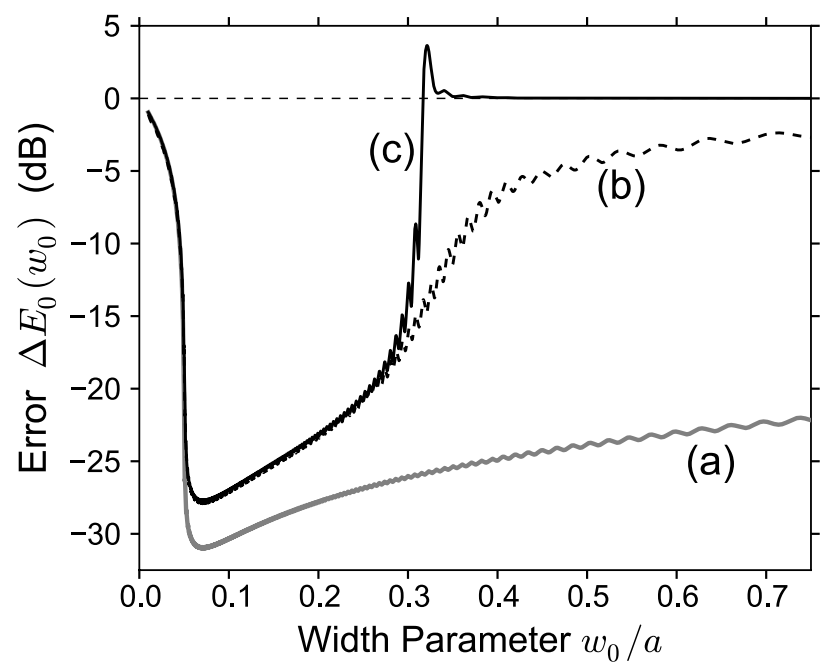

Fig. 7. Errors of the LG syntheses with varying width parameter, $w_{0}$, for a (a) circular aperture [8], (b) thin spherical lens, and (c) conical lens with Fresnel numbers $N_{F}=20$, using $M=199$. The optimal value of $w_{0}$ determined by Eq. (5) is $0.071 a$.

reconstruction error, $\Delta E_{0}$, in each case. Although $\Delta E_{0}$ increases with values of $w_{0}$ greater than that suggested by Eq. (5), maximal errors are not attained in the data shown for expansion of the spherical lens or basic aperture function, which, with low frequency features, can be partially reconstructed using larger values of $w_{0}$. A clear deviation occurs in the error calculations of the axicon's expansion, with the range of values of $w_{0}$ that permit acceptable synthesis becoming significantly reduced. In this case, a critical value of the width parameter occurs at $w_{0}=0.32 a$, corresponding to the value above which the highest radial frequency within the LG mode set, $\kappa_{M}(0)$ from Eq. (16), becomes less than the radial frequency, $\alpha$, of the axicon beam, therefore disallowing any appropriate synthesis. For an expansion of the axicon lens with smaller values of $M$, this critical value of $w_{0}$ will decrease toward that given by Eq. (므) with the latter subsequently losing its validity as an optimal parameter and the LG expansion becoming incapable of providing an appropriate synthesis. A similar feature will occur in expansion of the spherical lens.

Suggesting a general rule of thumb, the LG expansion using Eq. (5) is observed to remain suitable for synthesis of lens functions when the shifted or stretched LG spectra, see Fig. 6, are insignificantly truncated at $m=M$, i.e., $\mu+\Delta m<M$. This can be approximated with Eq. (17) for an axicon as

$$
M>\frac{\alpha a}{2},
$$

or for a thin spherical lens as

$$
M>\frac{k a^{2}}{2 f} .
$$

Simplified as $M>\pi N_{F}$, these conditions suggest the absolute minimum number of modes needed to 
simulate the basic geometric propagation features of a lens-modulated beam using the fundamental LG expansion as described in Eq. (4).

The relatively informal derivation of such rules of thumb is undesirable, but, in comparison with the more familiar and well-developed Fourier concepts of optics, the LG and related expansions lack accurate analytical descriptions for the varying spatial frequencies of their basis functions. In Siegman's derivation of a sampling theorem for the HG expansion of a field, which may potentially be modified for the LG technique, the HG functions are discussed as having a single frequency; an average value derived from the number of irregular oscillations across the estimated width of the functions [10]. Although enabling useful applications, such an approximation is suggestive of the somewhat imprecise nature of analysis involving the higher-order Gaussian functions.

\section{B. Optimization of Axial Field Calculations}

The optimization of aperture synthesis is a primary topic of interest in discussions of series expansion methods $[8,10,15]$. However, we are not interested in the synthesis of an aperture/lens structure per se, but more so its total diffractive influence on a propagating wave. Thus, any optimization of a series expansion for field calculations beyond an aperture must consider the important effects that are independent of the aperture's pupil function, i.e., the formation of boundary waves at its outer edge $[17,18,31]$. In Ref. [7] the convergence of the LG expansion to the FDI calculation was illustrated in the time domain for pulsed fields, but without indication of the number of modes required for suitable accuracy. Here, we can present a reliable prediction of the minimum value of $M$ needed for acceptable convergence of the LG expansion for on-axis fields, a previous statement of which is not known to the authors. This is given within a general discussion of the technique for CWs, but will have relevance in the simulation of pulses and, although illustrated for a conical lens, the described effects are generally relatable to all circularly symmetric apertures and lenses.

The high-frequency fluctuations of intensity typically observed in the on-axis structure of CW fields diffracted at an aperture are generated by the interference of the boundary waves originating at the edge of the aperture with the geometric wave that maintains the fundamental directional features of the (modulated) incident field [17,18,31,38], see Fig. 3(b). With a comparison to the FDI calculation,
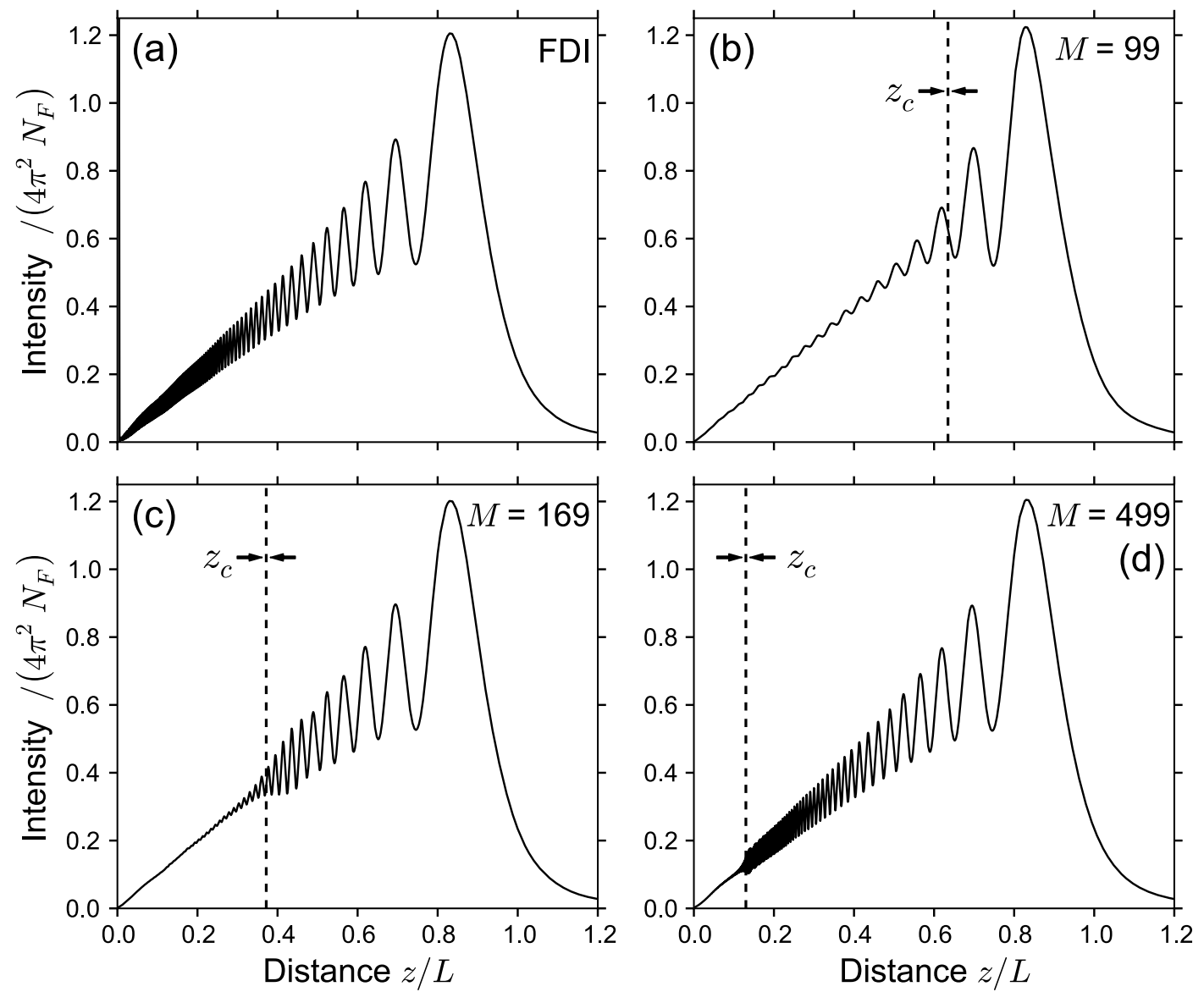

Fig. 8. Axial irradiance of a CW plane wave modulated by a $N_{F}=20$ conical lens, calculated using (a) the FDI, and (b)-(d) the LG expansions of various sizes. The dashed vertical lines represent the confocal plane, $z_{c}$, of the constituent modes, and the intensity values are normalized to the maximum value of Eq. (15). 
Fig. 8 depicts how the predicted axial irradiance of a CW plane wave modulated by an axicon is influenced by the truncation of a LG series to a finite number of beam modes.

Figure 8 illustrates clearly the effects of truncating a series expansion, with a loss of the highly oscillatory intensity structure in the near field. This is an inherent feature of such methods [2-5], see e.g. Fig. (4) of Ref. [4], and is due to the reduced ability of a truncated series calculation to represent the propagation features of the boundary waves at planes closer to the aperture. Assuming paraxial conditions, with the diffraction of a wave at an aperture at $z=0$ the highest radial frequency on the axis at any plane $z>0$ is that associated with the parabolic boundary wave that has a radial frequency at $r=0$ of $[\underline{7}, \underline{17}, \underline{18}]$

$$
k_{a}(z)=k a / z .
$$

Subsequently, for the axial characteristics of a boundary wave to be suitably represented by the LG expansion, the radial frequencies at the axis present within the finite set of propagating LG modes must replicate $k_{a}(z)$. To first state the condition under which such representation will occur, it is found that, with application of Eq. (5), the on-axis radial frequency of the mode with the highest index, $M$, $\kappa_{M}(z)$ from Eq. (16), exceeds that of the boundary wave at planes beyond the confocal range, $z_{c}$, with the LG series converging toward the complete axial prediction of the FDI in that region only. Representing the confocal range using $w_{0}$ from Eq. (5), the basic criterion for axial convergence of the $\mathrm{LG}$ series expansion at any plane, $z$, is therefore

$$
M>\frac{k a^{2}}{2 z}, \quad \text { i.e. } z>z_{c} .
$$

There is, however, a slight approximation in the simple derivation of the stated condition. With an adherence to Eq. (5), Eq. (20) is actually determined from the observation that the on-axis radial frequency of a fictitious LG mode with $m=M-1 / 2$ equates exactly with that of the boundary wave at $z=z_{c}$, i.e., from Eq. (16) with $w\left(z_{c}\right)=2^{1 / 2} w_{0}$,

$$
\kappa_{M-1 / 2}\left(z_{c}\right)=\frac{2}{\sqrt{2} w_{0}} \sqrt{2(M-1 / 2)+1}=2 \frac{M}{a}=k_{a}\left(z_{c}\right)
$$

with $\kappa_{M-1 / 2}(z)>k_{a}(z)$ at planes beyond $z_{c}$. Equivalent relations will hold for $\kappa_{M}$, with an adaptation of Eq. (5) to $w_{0}=a /(M+1 / 2)^{1 / 2}$, but the approximation $M \approx M-1 / 2$ has negligible effect in practical applications.

Equation (20) is proposed to be a useful and important parameter in the application of the LG expansion. For calculation of pulses with Gaussian spectra, assuming a constant value of $M$ for all frequency components, a suitable adaptation of Eq. (20) is $M>\pi(1+\gamma) N_{0}$, with $N_{0}=a^{2} /\left(\lambda_{0} z\right)$, which accounts for the higher field frequencies within a spectrum. This value can be seen to conform to the error analysis of the method's application for pulses shown in Fig. 11 of Ref. [7]. It is to be noted that the evolution of the expansion results toward complete convergence in $z>z_{c}$ is not an abrupt transition, as shown in Fig. 8, particularly with lower values of $M$.

\section{Pulsed Axicon Beams}

Figure 9 illustrates the propagation of a pseudo $X$ wave $[3 \overline{0}, 41-43]$ generated by axicon modulation of an ultrashort pulse using the LG expansion, with $L_{0}=L\left(\omega_{0}\right)$. The propagation of such fields generated using spatial light modulators has recently been described in Ref. [44].

Due to the separation of the geometric and boundary waves, see Fig. 9(a) or Fig. 3(b), a diffracted pulse's intensity will exhibit a less oscillatory variation than that of a CW in the same system $[18,45]$. Figure 10 illustrates the effect of this phenomena on the maximum instantaneous axial intensity, $|E|^{2}$, of a pulsed axicon beam at each plane. For $\gamma=$ 0.1 the intensity distribution shows a more oscillatory structure, due to the longer durations of the boundary and geometric pulses, which causes overlap and interference between the two features over

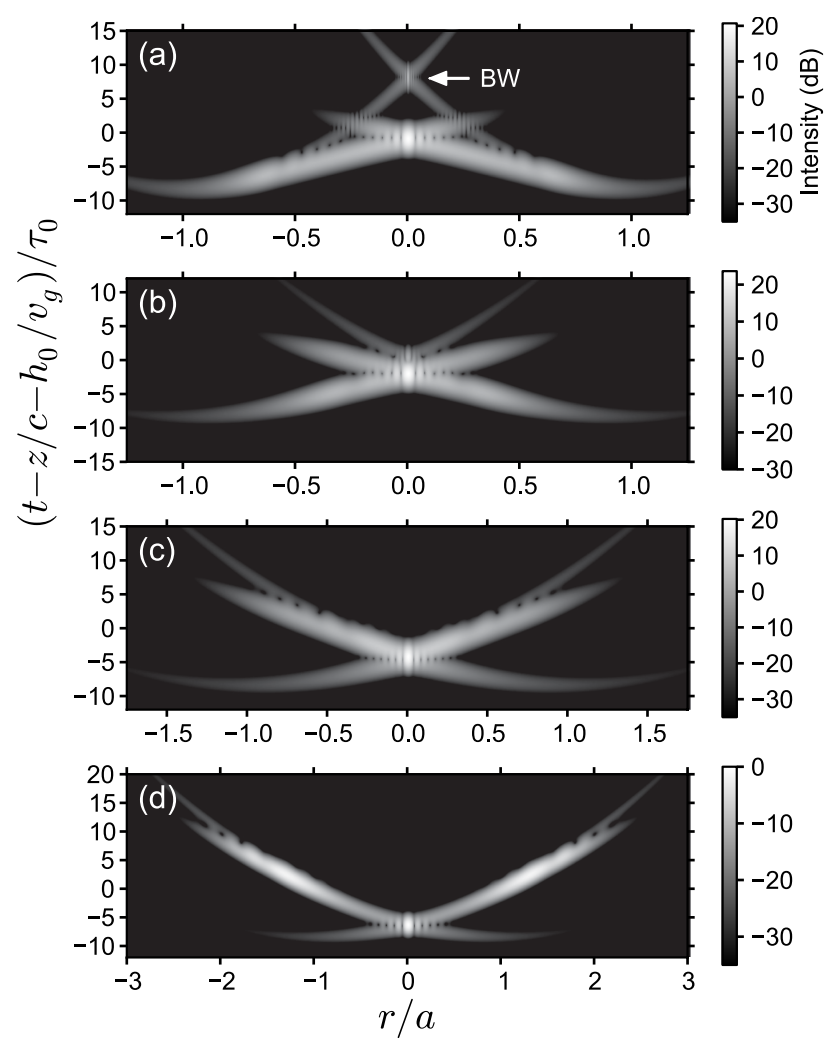

Fig. 9. Propagation of a $\gamma=0.3$ pulse modulated by a $N_{0}=12$ conical lens, using a LG expansion with $M=299$. The fields are simulated at $z=$ (a) $L_{0} / 4$, (b) $L_{0} / 2$, (c) $L_{0}$, (d) $2 L_{0}$. "BW" in (a) highlights the boundary wave. 

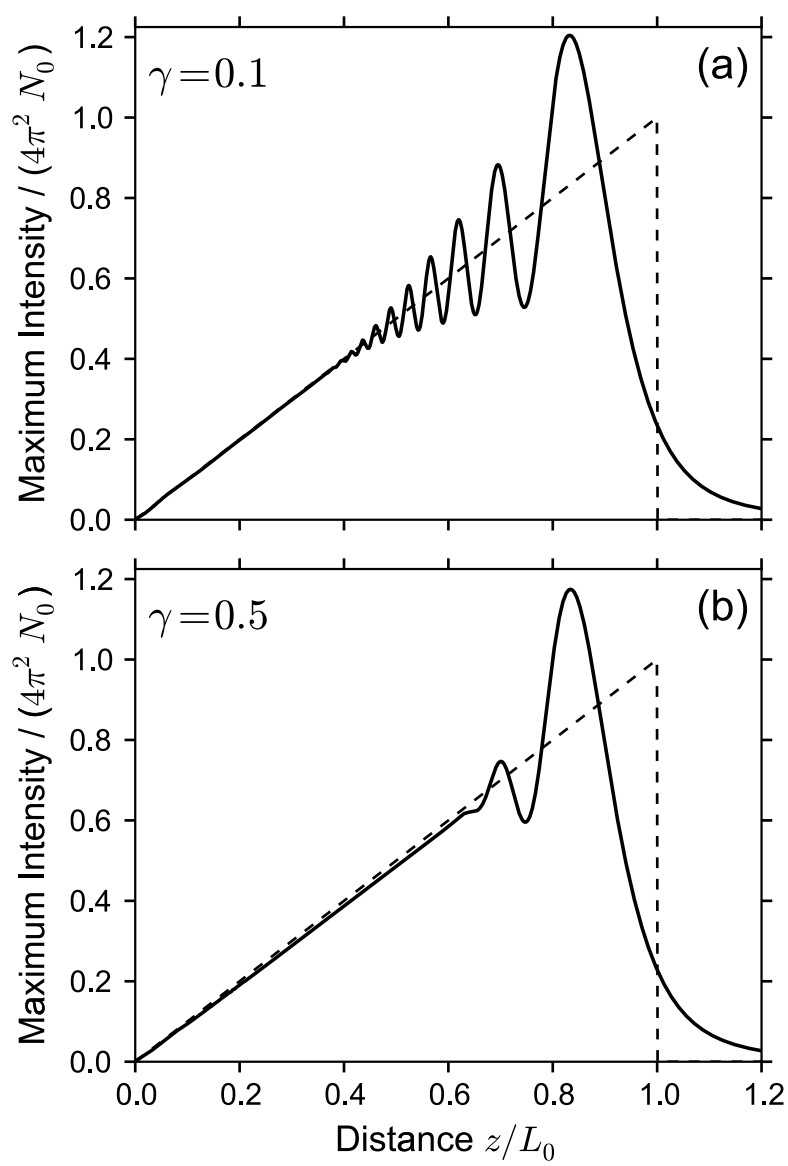

Fig. 10. Maximum instantaneous axial irradiance of a plane wave pulse refracted by a $N_{0}=20$ conical lens for two values of the field's fractional bandwidth, $\gamma$. The dashed lines represent the geometric prediction of the axial intensity from Eq. (15).

a greater distance than in the case of the much shorter pulse with $\gamma=0.5$. The limitations of the LG series method illustrated in Fig. $\underline{8}$ do not interfere in the specific calculations of maximum instantaneous intensity shown in Fig. 10. In this case, the most intense feature of the field is the geometric wave that can be suitably reconstructed at all planes. However, complete calculation of the corresponding timeaveraged intensity will require appropriate representation of the boundary wave and, for that scenario, the series expansions may exhibit limitations related to those shown in Fig. 8 .

Without knowledge of appropriate analytical expressions, the application of an efficient and adaptable numerical technique is particularly beneficial in calculating the significant amount of data required for the generation of Fig. 10 .

\section{Conclusion}

The LG expansion has been described as an efficient complement to the existing techniques for simulation of pulses modulated by refractive and diffractive lenses. The $(1+1) \mathrm{D}$ field images shown here were calculated with a maximum of $1001 \times 1001$ points, using 201 samples of a discrete Fourier integral in Eq. (7). With basic custom code, this required approximately $90 \mathrm{~min}$ on a standard portable computer when using a value of $M=499$. However, such resolution is obviously not always essential and can be significantly reduced to enable the procedure's application as an efficient research tool, allowing for impromptu examination of the propagation features of pulses modulated by nonstandard lenses and gratings. The inherent efficiency of the method's formulation as a sum, augmented with vector and matrix programming methods, may be considered as a trade-off with the loss of complete convergence toward the FDI results, as described in Section 3.B, but the importance of those inevitable limitations can be dependent on the specific application. Although directly related to axial calculations, the criterion for convergence given by Eq. (20) can also be applied as a helpful rule in the simulation of $(1+1) \mathrm{D}$ pulse structures in the near field. However, at distances further from the aperture the radial frequencies at the optical axis decrease and become comparable in magnitude to those at off-axis points, which will require the application of larger mode sets than suggested by Eq. (20).

The LG expansion was applied here with exclusive reference to the symmetric cylindrical regime, but the technique can be adapted for nonsymmetric or obliquely propagating fields using the rotationally variant associated LG beam modes, allowing for analysis of lens aberrations [14]. The HG beam modes can be applied for a 2D Cartesian description of an aperture in a similar manner, allowing for greater variation in the asymmetry of fields that can be represented [46].

\section{References}

1. J. W. Goodman, Introduction to Fourier Optics, 2nd ed. (McGraw-Hill, 1996).

2. J. J. Wen and M. A. Breazeale, "A diffraction beam field expressed as the superposition of Gaussian beams," J. Acoust. Soc. Am. 83, 1752-1756 (1988).

3. D. Ding and Y. Zhang, "Notes on the Gaussian beam expansion," J. Acoust. Soc. Am. 116, 1401-1405 (2004).

4. M. Zamboni-Rached, E. Recami, and M. Balma, "A simple and effective method for the analytic description of important optical beams, when truncated by finite apertures," Appl. Opt. 51, 3370-3379 (2012).

5. M. Zamboni-Rached, K. Z. Nóbrega, and C. A. Dartora, "Analytic description of Airy-type beams when truncated by finite apertures," Opt. Express 20, 19972-19977 (2012).

6. R. J. Mahon and J. A. Murphy, "Gaussian beam mode analysis of optical pulses," Proc. SPIE 8171, 81710H (2011).

7. R. J. Mahon and J. A. Murphy, "Diffraction of an optical pulse as an expansion in ultrashort orthogonal Gaussian beam modes," J. Opt. Soc. Am. A 30, 215-226 (2013).

8. R. Borghi, F. Gori, and M. Santarsiero, "Optimization of Laguerre-Gauss truncated series," Opt. Commun. 125, 197-203 (1996)

9. B. D. Cook and W. J. Arnoult III, "Gaussian-Laguerre/Hermite formulation for the nearfield of an ultrasonic transducer," J. Acoust. Soc. Am. 59, 9-11 (1976).

10. A. E. Siegman, Lasers (University Science, 1986).

11. J. A. Murphy and A. Egan, "Examples of Fresnel diffraction using Gaussian modes," Eur. J. Phys. 14, 121-127 (1993).

12. E. Cagniot, M. Fromager, and K. Ait-Ameur, "Modeling the propagation of apertured high-order Laguerre-Gaussian beams by a user-friendly version of the mode expansion method," J. Opt. Soc. Am. A 27, 484-491 (2010). 
13. J. A. Murphy, "Examples of circularly symmetric diffraction using beam modes," Eur. J. Phys. 14, 268-271 (1993).

14. N. Trappe, J. A. Murphy, and S. Withington, "The Gaussian beam mode analysis of classical phase aberrations in diffraction-limited optical systems," Eur. J. Phys. 24, 403412 (2003).

15. Y. Liu and B. Lü, "Truncated Hermite-Gauss series expansion and its application," Optik 117, 437-442 (2006).

16. R. L. Phillips and L. C. Andrews, "Spot size and divergence for Laguerre Gaussian beams of any order," Appl. Opt. 22, 643-644 (1983).

17. Z. L. Horváth and Z. Bor, "Diffraction of short pulses with boundary diffraction wave theory," Phys. Rev. E 63, 026601 (2001).

18. O. Mendoza-Yero, G. Mínguez-Vega, J. Lancis, M. FernándezAlonso, and V. Climent, "On-axis diffraction of an ultrashort light pulse by circularly symmetric hard apertures," Opt. Express 15, 4546-4556 (2007).

19. Ó. Martínez-Matos, P. Vaveliuk, J. G. Izquierdo, and V. Loriot, "Femtosecond spatial pulse shaping at the focal plane," Opt. Express 21, 25010-25025 (2013).

20. J. Alda, J. M. Rico-Garcia, J. M. Lopez-Alonso, B. Lail, and G. Boreman, "Design of Fresnel lenses and binary-staircase kinoforms of low value of the aperture number," Opt. Commun. 260, 454-461 (2006).

21. D. W. Prather, D. Pustai, and S. Shi, "Performance of multilevel diffractive lenses as a function of F-number," Appl. Opt. 40, 207-210 (2001)

22. Y. Zhang, J. Chen, and X. Yea, "Multilevel phase Fresnel zone plate lens as a near-field optical element," Opt. Commun. 269, 271-273 (2007).

23. D. Feng, P. Ou, L.-S. Feng, S.-L. Hu, and C.-X. Zhang, "Binary sub-wavelength diffractive lenses with long focal depth and high transverse resolution," Opt. Express 16, 20968-20973 (2008).

24. S. Wang and X.-C. Zhang, "Pulsed terahertz tomography," J. Phys. D 37, R1-R36 (2004).

25. M. Kempe, U. Stamm, B. Wilhelmi, and W. Rudolph, "Spatial and temporal transformation of femtosecond laser pulses by lenses and lens systems," J. Opt. Soc. Am. B 9, 1158-1165 (1992).

26. D. Deng, H. Guo, D. Han, M. Liu, and C. Li, "Effects of dispersion and longitudinal chromatic aberration on the focusing of isodiffracting pulsed Gaussian light beam," Phys. Lett. A 334, 73-80 (2005)

27. Z. Bor, "Distortion of femtosecond laser pulses in lenses and lens systems," Opt. Lett. 14, 119-121 (1989).

28. P. Bowlan, P. Gabolde, and R. Trebino, "Directly measuring the spatio-temporal electric field of focusing ultrashort pulses," Opt. Express 15, 10219-10230 (2007).

29. O. Mendoza-Yero, B. Alonso, O. Varela, G. Mínguez-Vega, Í. J. Sola, J. Lancis, V. Climent, and L. Roso, "Spatio-temporal characterization of ultrashort pulses diffracted by circularly symmetric hard edge apertures: theory and experiment," Opt. Express 18, 20900-20911 (2010).
30. P. Bowlan, H. Valtna-Lukner, M. Lõhmus, P. Piksarv, P. Saari, and R. Trebino, "Measuring the spatiotemporal field of ultrashort Bessel-X pulses," Opt. Lett. 34, 2276-2278 (2009).

31. P. Saari, P. Bowlan, H. Valtna-Lukner, M. Lõhmus, P. Piksarv, and R. Trebino, "Basic diffraction phenomena in time domain," Opt. Express 18, 11083-11088 (2010).

32. M. Lõhmus, P. Bowlan, P. Piksarv, H. Valtna-Lukner, R. Trebino, and P. Saari, "Diffraction of ultrashort optical pulses from circularly symmetric binary phase gratings," Opt. Lett. 37, 1238-1240 (2012).

33. P. Piksarv, P. Bowlan, M. Lõhmus, H. Valtna-Lukner, R. Trebino, and P. Saari, "Diffraction of ultrashort Gaussian pulses within the framework of boundary diffraction wave theory," J. Opt. 14, 015701 (2012).

34. C. J. Zapata-Rodríguez, "Temporal effects in ultrashort pulsed beams focused by planar diffracting elements," J. Opt. Soc. Am. A 23, 2335-2341 (2006).

35. S. Zwick, C. Schaub, T. Haist, and W. Osten, "Light fields with an axially expanded intensity distribution for stable threedimensional optical trapping," Opt. Express 18, 1994119950 (2010)

36. N. Trappe, R. Mahon, W. Lanigan, J. A. Murphy, and S. Withington, "The quasi-optical analysis of Bessel beams in the far infrared," Infrared Phys. Technol. 46, 233-247 (2005).

37. C. J. Zapata-Rodríguez and A. Sánchez-Losa, "Threedimensional field distribution in the focal region of lowFresnel-number axicons," J. Opt. Soc. Am. A 23, 3016-3026 (2006).

38. A. Burvall, P. Martinsson, and A. T. Friberg, "Communication modes applied to axicons," Opt. Express 12, 377-383 (2004).

39. J. A. Monsoriu, W. D. Furlan, P. Andrés, and J. Lancis, "Fractal conical lenses," Opt. Express 14, 9077-9082 (2006).

40. J. Durnin, J. J. Miceli, Jr., and J. H. Eberly, "Nondiffracting beams," Phys. Rev. Lett. 58, 1499-1501 (1987).

41. M. Zamboni-Rached, "Localized superluminal solutions to the wave equation in (vacuum or) dispersive media, for arbitrary frequencies and with adjustable bandwidth," Opt. Commun. 226, 15-23 (2003).

42. P. Saari and H. Sõnajalg, "Pulsed Bessel beams," Laser Phys. 7, 32-39 (1997)

43. J. Lloyd, K. Wang, A. Barkin, and D. Mittleman, "Characterisation of apparent superluminal effects in the focus of an axicon lens using terahertz time-domain spectroscopy," Opt. Commun. 219, 289-294 (2003).

44. L. Froehly, M. Jacquot, P. A. Lacourt, J. M. Dudley, and F. Courvoisier, "Spatiotemporal structure of femtosecond Bessel beams from spatial light modulators," J. Opt. Soc. Am. A 31, 790-793 (2014).

45. M. Gu and X. S. Gan, "Fresnel diffraction by circular and serrated apertures illuminated with an ultrashort pulsed-laser beam,” J. Opt. Soc. Am. A 13, 771-778 (1996).

46. R. May, J. A. Murphy, C. O'Sullivan, M. Gradziel, and N. Trappe, "Gaussian beam mode analysis of phase gratings," Proc. SPIE 6893, 68930G (2008). 